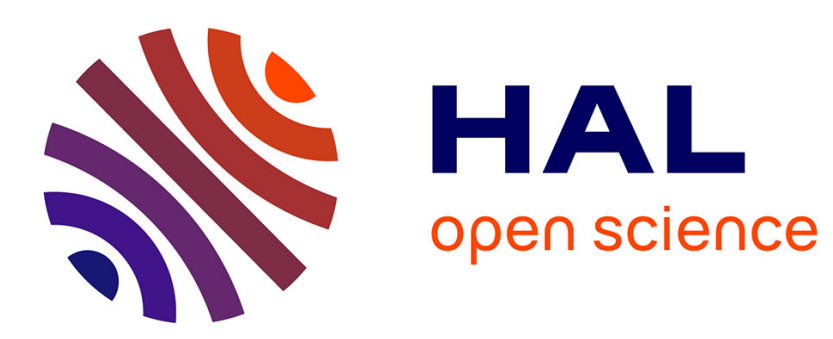

\title{
Formes symboliques et émergence de valeurs; Pour une cognition culturalisée
}

Jean Lassègue

\section{To cite this version:}

Jean Lassègue. Formes symboliques et émergence de valeurs; Pour une cognition culturalisée. 2004. halshs-00003271

\section{HAL Id: halshs-00003271 \\ https://shs.hal.science/halshs-00003271}

Preprint submitted on 15 Nov 2004

HAL is a multi-disciplinary open access archive for the deposit and dissemination of scientific research documents, whether they are published or not. The documents may come from teaching and research institutions in France or abroad, or from public or private research centers.
L'archive ouverte pluridisciplinaire HAL, est destinée au dépôt et à la diffusion de documents scientifiques de niveau recherche, publiés ou non, émanant des établissements d'enseignement et de recherche français ou étrangers, des laboratoires publics ou privés. 


\title{
Formes symboliques et émergence de valeurs
}

\section{Pour une cognition culturalisée}

\author{
Jean Lassègue \\ Laboratoire Lattice - UMR 8094 \\ Ecole Normale Supérieure \\ 1 rue Maurice Arnoux \\ F-92120 Montrouge \\ jean@lassegue.net
}

RÉSUMÉ. On essaye de caractériser un niveau général d'intelligibilité du sens - celui de la "forme symbolique " - dans un système d'interaction sociale où l'on ne présuppose pas l'individualité préalable des agents. Ce niveau général d'intelligibilité est caractérisé par cinq traits saillants dont le rôle n'est pas cantonné à une sphère particulière de l'expérience. On récuse l'idée que ces traits soient identifiables à une table des catégories classique, dans la mesure où leur rôle, dans chaque transaction particulière, n'est pas fixé une fois pour toutes.

MOTS-CLÉS : formes symboliques, émergence de valeurs, catégorie, transcendantal, Cassirer. 


\section{Introduction ${ }^{1}$}

Plaçons-nous d'emblée dans un cadre non mentaliste au sein duquel les interactions entre les individus fondent leurs identités. Essayons, dans ce cadre, de rendre compte de l'émergence de valeurs sémiotiques partagées sans présupposer leur existence préalable.

Si l'on renonce à chercher des invariants assimilés à des principes génératifs universels qui seraient immédiatement projetés en tant que fonctions cognitives supérieures chez les individus mais que l'on ne veuille pas non plus se limiter à la pure description des jeux indéfiniment changeants dans lesquels ces valeurs sémiotiques apparaissent au sein des interactions, bref, si l'on veut avoir un point de vue un tant soit peu général sur la construction des valeurs sémiotiques, on doit considérer que ce qui fait valeur provient non pas d'un invariant obtenu par abstraction hors de tout domaine d'interaction mais d'une flexibilité trans-domaniale de ces valeurs perdurant à travers différents contextes d'activité.

Cette approche permet de modifier le rapport entre invariant et variabilité. En effet, si l'invariant n'est plus pensé comme le résultat d'une abstraction hors de tout contexte d'interaction mais comme flexibilité, sa stabilité exige de passer à travers de multiples domaines pour conserver son identité. La variabilité n'est donc pas ce qui s'oppose à la stabilité de l'invariant mais au contraire ce qui l'accomplit au cours d'un processus : l'invariant se définit alors par sa capacité à se prêter à la différenciation. On doit donc s'attacher à l'étude des conditions de stabilisation trans-domaniale de valeurs au sein d'activités dont la diversité est nécessaire à la mise au jour de cette stabilité trans-domaniale. On rejette pour ce faire l'idée de principes génératifs universels entièrement encapsulés dans la cognition individuelle pour ouvrir au contraire la cognition à une interaction avec un environnement qui rend possible l'individuation. On s'éloigne donc de tout idée de pré-programmation (génétique, algorithmique, symbolico-représentationnelle) susceptible de calculer d'avance une trajectoire comportementale ou cognitive limitée à un individu abstrait de son environnement, même si l'on ne récuse pas l'idée de programme, entendu comme simple phase dans un processus complexe ou l'idée d'un programme d'action, cognitivement représentable par les individus au cours de leurs interactions.

C'est cette stabilisation trans-domaniale de valeurs à travers des activités multiples que l'on cherche à décrire par l'expression de « forme symbolique ».

\footnotetext{
1. Cet article est le résultat d'un travail mené depuis plusieurs années en collaboration avec Yves-Marie Visetti au sein d'un certain nombre de groupes de travail, tout particulièrement dans le cadre du séminaire « Formes Symboliques » que je co-dirige avec lui.
} 


\section{Formes symboliques}

Un rappel historique tout d'abord. L'expression de « forme symbolique » est due à Ernst Cassirer. On la trouve en particulier dans son ouvrage majeur, La philosophie des formes symboliques (1923-1929) : l'usage du pluriel montre qu'il considérait comme nécessaire la multiplicité de ces formes, renvoyant à des activités sémiotiques ayant chacune leur régime de sens intrinsèque. Chez lui, trois formes symboliques sont considérées comme canoniques : le langage, le mythe et la science (d'où les titres des trois tomes publiés de son livre). Mais ces régimes de sens, tout en ayant leur identité propre, ne sont pas exclusifs les uns des autres : par exemple, le langage et le mythe ont une proximité telle qu'il est difficile de les distinguer radicalement (Cassirer a même montré leur profonde parenté dans un autre livre, Langage et mythe, et a fait de leur divorce - provisoire selon lui - l'acte de naissance de la rationalité scientifique et philosophique en Grèce classique). Le point de vue de Cassirer a ceci de « cognitif» (ce n'est pas son vocabulaire) qu'il cherche à rendre compte de la construction du transcendantal, conçu comme capacité anticipatrice, à travers les différents régimes de sens que sont les formes symboliques. On verra plus bas en quoi cette construction implique d'emblée une culturalisation du cognitif.

Ce point d'histoire éclairci, revenons à la caractérisation des formes symboliques. Elles sont des passages obligés qui visent à stabiliser des valeurs associées à des objets de transaction. La tractation sur la valeur s'opère sur des objets de transaction : par « objet de transaction » il faut entendre la façon dont un support matériel est investi par la valeur à tous les niveaux où peut se glisser un écart différentiel. Cela peut être un matériau phonique (les phonèmes d'une langue), un matériau écrit (la monnaie frappée, l'écriture), mais aussi un « matériau » humain (l'esclave assimilé à un outil par rapport à l'homme libre, la jeune fille nubile par rapport à la femme mariée ou les enfants à initier). Par exemple, une pièce de monnaie matérialise dans des marques (que ce soit un certain métal, une certaine forme, un certain poinçon, un certain papier, etc.) la possibilité de concevoir une échelle de valeur. Ces marques matérielles ne sont pas des représentations amorphes de la valeur mais son expression car, sans elles, la valeur resterait trop volatile pour faire l'objet d'une transaction. Deux caractéristiques sont particulièrement importantes concernant l'expression de la valeur au moyen d'un support matériel. D'une part, ce caractère matériel introduit une diversité intrinsèque dans les marques matérialisant la valeur (par exemple, dans le cas de la monnaie : forme, poids, poinçon, etc.). D'autre part, cette stabilisation des valeurs sur des objets de transactions est socialement instituée : pour reprendre l'exemple monétaire, on voit bien que la spécificité matérielle d'un certain support est le fruit d'une institution qui reste toujours implicitement présente dans chaque transaction et sert de garantie à son bon déroulement (la pièce doit avoir telle ou telle forme, tel ou tel poids, tel ou tel poinçon pour être acceptée comme jouant le rôle de support de valeur). De même, l'esclave n'est esclave que par rapport à une institution qui le décrète et est en mesure de faire respecter son décret. Mais cette institution de la valeur n'est pas 
nécessairement intentionnelle, comme le montre abondamment le cas du langage où d'apparentes régularités dans la construction du sens ne proviennent pas de l'instanciation de règles abstraites mais sont en fait le fruit d'une réélaboration postérieure de type analogique ${ }^{2}$.

Ainsi les interactions entre individus se stabilisent-elles autour de passages obligés culturellement institués - les formes symboliques - qui orientent ces interactions selon des modalités diverses. L'exemple des différents régimes de parenté, définissant des partenaires sexuels interdits, permis, obligés ou préférés est particulièrement éclairant : des règles, explicites ou tacites, orientent et façonnent le comportement sexuel en en faisant une forme instituée, proprement culturelle. Du point de vue individuel, ces passages obligés que sont les formes symboliques apparaissent alors comme des anticipations de la forme de toute interaction possible qui contraignent la façon dont les interactions inter-individuelles peuvent se produire : elles ont statut de normes. Ainsi les formes symboliques permettent-elles de révéler les tendances générales des interactions humaines et de définir de grands types d'activité culturelle. Aux trois formes canoniques décrites originellement par Cassirer (langage, mythe, science), il est possible d'en ajouter d'autres (certaines seulement mentionnées par Cassirer) de nature esthétique (écoles, styles, tendances), politique (règles matrimoniales, codes juridiques, formes de l'autorité), économique (types d'échange, types de biens, types de travail) ou rituelle (religions, festivités, techniques).

Cette présentation très générale de la nature et du rôle attribué aux formes symboliques pose un certain nombre de problèmes, dont trois me paraissent particulièrement importants dans la mesure où ils abordent la question de la construction d'une forme symbolique ainsi que le rapport entre formes symboliques : peut-on caractériser toutes les formes symboliques à partir de traits communs relativement stables? Peut-on énumérer ces formes en en dressant une typologie? Comment les formes symboliques interagissent entre elles tout en conservant leurs identités? Je vais essayer d'esquisser successivement des réponses à ces trois questions.

2. Comme le fait remarquer Saussure : «En français, on a dit longtemps : il preuve, nous prouvons, ils preuvent. Aujourd'hui on dit il prouve, ils prouvent, formes qui ne peuvent s'expliquer phonétiquement; il aime remonte au latin amat; tandis que nous aimons est analogique pour amons. [...]. C'est l'école néo-grammairienne qui a pour la première fois assigné à l'analogie sa vraie place en montrant qu'elle est, avec les changements phonétiques, le grand facteur de l'évolution des langues, le procédé par lequel elles passent d'un état d'organisation à un autre ». Cours de linguistique générale, Payot, Paris, p. 222-223. 


\section{Peut-on caractériser toutes les formes symboliques à partir de traits communs relativement stables?}

Telle qu'elle est posée, la question pourrait suggérer l'existence d'un invariant décontextualisé rendant compte de la dynamique propre aux formes symboliques et qui s'instancierait selon divers paramétrages locaux. Mais cette approche risquerait de compromettre l'idée même de la diversité des activités nécessaire à la constitution d'une forme symbolique ainsi que la diversité des rapports des formes symboliques entre elles. Il faut donc être attentif aux parcours de construction des formes dans leur diversité avant de rechercher des invariants hors contexte ${ }^{3}$ : les traits propres aux interactions culturelles en général, en tant qu'elles sont médiatisées par une forme symbolique, se laissent approcher en tant que capacités de différenciation et non en tant qu'instanciations d'un type. Cinq traits sont particulièrement saillants. Par souci de clarté, j'en donne à chaque fois une description rapide.

\subsection{Trans-domanialité}

Les formes symboliques ne sont jamais localisées au point d'être cantonnées à un domaine d'activité particulier.

Autrement dit, les valeurs associées aux résultats des interactions sociales ne sont pas limitées au champ particulier propre à cette interaction. Prenons un cas esthétique : les historiens de l'art ont montré que des formes esthétiques perduraient à travers la multiplicité des écoles et des styles. Par exemple, il est possible de retrouver dans la statuaire de la Renaissance un schème hérité de la statuaire antique sous une autre appellation (la représentation d'Hercule devient celle d'un saint). De même, une forme esthétique quelconque ne se limite pas au champ de l'esthétique proprement dit mais le déborde sous la forme de tendances esthétiques, dans l'artefact, le vêtement ou le théorème. On peut en tirer deux conséquences.

D'une part, la stabilité d'une valeur matérialisée dans un objet de transaction ne dépend pas seulement d'une transaction particulière mais participe d'un champ de transactions plus global. Par exemple, la fixation d'un prix dépend certes d'une transaction particulière mais aussi d'un marché où d'autres critères entrent en considération (offre/demande; vente en gros/au détail, vente autorisée/vente interdite, etc.). C'est le différentiel entre une transaction particulière et le champ de transaction plus global qui est généralement inaccessible à la conscience des individus mais qui contraint cependant leurs interactions (le cas de la grammaire,

3. Les listes de traits anthropologiques dégagés par Hocart après une recherche concernant des civilisations et des périodes historiques multiples, sont, à cet égard, exemplaires (cf. Kingship, Oxford University Press, Oxford, 1927, chap. 7 et Social Origins, Watts \& Co, Londres, 1954, chap. 4). 
généralement inaccessible aux locuteurs de la langue - sauf aux linguistes professionnels ! - est un cas typique).

D'autre part, d'un point de vue épistémologique, on doit remarquer que le schéma de la causalité classique (liée, à l'origine, à la physique dite classique) est inopérant pour rendre compte de l'émergence et de la stabilisation d'une valeur : une forme symbolique ne produit pas causalement des valeurs parce qu'il lui faudrait être limitée à une région spécifique de l'activité humaine pour que la relation de cause à effet puisse être correctement isolée. Réciproquement, aucune activité ne peut monopoliser intégralement une forme symbolique, qui déborde la région particulière d'activité où elle possède le plus de prégnance, c'est-à-dire où son contenu sémiotique a été le plus travaillé pour lui-même.

\subsection{Transmissibilité}

Les formes symboliques sont des engagements pratiques ritualisés, hérités à travers le temps et possédant un pouvoir anticipateur.

Une forme symbolique ouvre donc à une temporalité spécifique en renvoyant à la fois à un héritage et à un avenir : toute interaction présente s'effectue sous l'égide de formes symboliques anticipatrices non-explicitement présentes, dont on hérite collectivement. Généralement, l'origine d'une forme symbolique particulière est rapportée à un passé immémorial (tous les mythes d'origine du langage, des dieux, de l'homme ou de la femme sont de ce type), dont la médiation peut être matérialisée sous l'aspect d'entités réelles ou imaginaires (totem, emblème, marques d'appartenance diverses). La validité des transactions sous l'égide de cette forme est projetée sur la totalité du futur ( «à la fin des temps », dit l'expression qui nous est familière). Remarquons que pour qu'il y ait transmission, il faut que des moments de transmission soient explicites : la façon de copier ou de réciter les textes sacrés, de battre monnaie, de prier, etc. doit faire l'objet d'une ritualisation explicite des gestes pendant des moments de vigilance collective, marqués comme tels. Ce sont ces moments qui relancent périodiquement la construction d'un sens en commun et qui forment l'objet même des rituels dont l'aspect trans-générationnel (souvent lié aux étapes d'une initiation) est capital. Cet aspect trans-générationnel met indirectement en scène la différence des sexes (conçue comme facteur biologique, comme institution sociale et comme modèle d'intelligibilité) puisque c'est par elle que transite l'idée même de génération.

\subsection{Tiers-terme}

Toute transaction se fait sous l'égide d'un tiers-terme considéré comme la source distante ou absente par rapport à laquelle l'interaction gravite. 
Pour éviter une régression indéfinie dans la confiance réciproque que doivent se témoigner par avance ceux qui inter-agissent, l'attention est focalisée vers un tiersterme qui joue le rôle de garant des transactions en général. Ce tiers-terme est dans la position d'un géométral : il résume en lui les différents points de vue sur la même interaction en anticipant sur l'aspect nécessairement situé attribué au rôle de chaque protagoniste. Le contrôle sur le tiers-terme tel qu'il est institué dans une société particulière permet ainsi de rendre compte des notions d'autorité et de pouvoir qui sont des anticipations de la forme que doit prendre l'interaction à venir.

Le tiers-terme peut prendre des formes diverses : figures totémiques ou divines, ancêtres, prohibitions, banques et institutions en général. Une mention particulière doit être faite concernant le sens linguistique, qui, lui aussi, relève du tiers-terme au sens employé ici. L'interaction verbale s'effectue en effet par rapport à un sens linguistique dont on suppose l'existence en soi (préalable à la transaction présente) et dont le contrôle est censé rendre possible une anticipation des transactions à venir ayant le sens linguistique pour objet.

\subsection{Opacité}

Il y a une opacité originelle propre aux formes symboliques en tant qu'elles sont instituées.

Cela vient du fait que, dans un modèle d'interaction qui ne présuppose pas une individuation préalable des agents, la construction des valeurs ne s'opère pas selon un schéma contractualiste ou utilitariste qui serait clairement représentable ou qui devrait d'abord se représenter clairement préalablement à l'interaction. L'opacité vient de ce que ni le désir ni les obligations réciproques entre agents ne sont fixées d'avance mais surtout que les moyens sémiotiques d'exprimer le désir ou les obligations en les fixant sur un support ne sont pas d'emblée en possession des agents : ces moyens sont concentrés dans les formes symboliques en tant qu'elles sont des institutions. Les institutions apparaissent alors comme ce qui permet de réunir des agents dont les rôles ne sont pas clairement fixés d'avance, même s'ils le deviennent au cours de l'interaction. L'exemple le plus frappant d'une telle opacité est celui des rituels : ce que requiert un rituel de la part d'un agent, c'est avant tout de posséder une disponibilité qui ultérieurement sera monnayée en efficacité dans une activité, en intelligibilité du monde ou en appartenance à un groupe mais qui ne les requiert pas au préalable.

\subsection{Auto-évaluation}

Toute interaction suscite sa propre évaluation, explicite ou implicite, sur une échelle de conformité et selon une norme explicitable. 
L'explicitation de la norme ne se fait pas par référence à une valeur objective dont on pourrait mesurer extérieurement la pertinence en la projetant sur une échelle d'avantages (fitness écologique, cognitive, sociale ou autre). C'est plutôt la différence temporelle entre l'aspect anticipateur des formes symboliques et la transaction particulière ici et maintenant qui fait surgir la norme en tant que procédure d'évaluation. La norme n'agit donc pas comme une catégorie abstraite parce qu'objective mais seulement comme ce qui induit évaluation. Si l'on reprend l'exemple de l'analogie décrit par Saussure, on voit bien que la construction de nombreuses régularités dans la langue relève de ce type de procédure.

Remarquons également - autre effet anticipateur de la norme-que son explicitation contient virtuellement sa capacité de transgression puisqu'en devenant consciente chez les agents, elle rend possible sa propre modification. C'est ce qui renforce la prise de conscience de la relative interchangeabilité des rôles dans l'interaction.

\section{Peut-on énumérer les formes symboliques en en dressant une typologie?}

Telle qu'elle est posée, la question revient à se demander s'il est possible de dresser une fois pour toutes une liste des formes symboliques qui serait comparable à une table des catégories. Puisque c'est à la mathématique que revient le rôle structurant rendant cohérent le domaine de la détermination catégorielle en général, le problème revient en fait à celui de la place que l'on entend accorder à l'objectivité mathématique dans la description des phénomènes culturels.

Si l'on se réfère à l'optique kantienne (qui fut le point de départ de Cassirer), on voit que l'accroissement progressif du champ de l'objectivité à des domaines phénoménaux nouveaux passe par une mathématisation appropriée aux domaines en question : en extension, à chaque nouveau domaine phénoménal doit correspondre une nouvelle modalité de la forme mathématique qui en est la condition transcendantale ; en compréhension, c'est par leur mathématique que des domaines réputés hétérogènes ont une parenté structurale assurant une cohérence objective globale à la science. C'est par exemple ainsi que J. Petitot interprète la théorie thomienne des catastrophes : il y voit la forme mathématique adéquate permettant de rendre compte de l'émergence des phénomènes de type structural dans les sciences humaines et sociales, ce qui permet de réintégrer dans le champ global de la mathesis des pans entiers de quasi-phénomènes qui n'avaient jusqu'alors pas reçu les conditions transcendantales adéquates pour accéder à une objectivité (c'était par exemple le cas de la notion de phonème ${ }^{4}$ ). Les formes symboliques relèvent-elles de cette optique sur la mathématisation?

Si c'était le cas, il faudrait renoncer au caractère d'emblée trans-domanial de ces formes qui ne sont pas suffisamment locales pour laisser à une générativité

4. J. Petitot, Les catastrophes de la parole, Maloine, Paris, 1985. 
mathématique le soin de les relier structuralement à la mathesis. Il faudrait en effet qu'une forme conçue comme locale puisse être étendue globalement au moyen d'une générativité mathématique adéquate. Or si les formes symboliques rendent possible une objectivité dans le cas où elles sont travaillées dans cette direction, leur intelligibilité générale n'est cependant pas dépendante de ce régime particulier du sens. Deux aspects doivent donc être pris simultanément en compte dans le cas des formes symboliques : d'une part, elles peuvent se mathématiser; d'autre part, leur intelligibilité ne dépend pas de leur mathématisation possible. Aussi la " difficulté » (pour qui se place dans l'optique kantienne tout au moins) liée à une intégration des formes symboliques au sein de la mathesis vient-elle de ce que ces dernières ne négocient pas le rapport entre le local et le global d'une manière telle qu'elle puisse s'inscrire dans une perspective transcendantale. Si l'on se reporte d'ailleurs à la façon dont Cassirer avait originellement posé le problème, on voit que celui-ci avait comparé la description générale des formes symboliques à une grammaire ${ }^{5}$ et non à une logique, indiquant sans doute par là que la strate de généralisation qu'il avait en vue (celle que $\mathrm{j}$ 'appelais un peu grossièrement en commençant la « construction du transcendantal ») n'était pas directement assimilable à la perspective kantienne que Kant rapporte à une logique. Il n'y a donc sans doute pas moyen de dresser une typologie des formes symboliques sous la forme d'une table des catégories, sauf à considérer un certain état de la science comme le terminus ad quem de toute évolution culturelle et non pas comme une option culturelle, historiquement situable (ce qui permet précisément de poser le problème de son objectivité6).

\section{Comment les formes symboliques interagissent entre elles tout en conservant leurs identités?}

Le problème que nous rencontrons est donc celui de la multiplicité des formes symboliques, de leur intrication réciproque et de leur éventuelle indépendance. Là encore, il semble qu'il n'y ait pas de réponse a priori susceptible de devancer la façon dont les rapports entre formes symboliques se tissent et que le recours à l'histoire soit indispensable pour faire l'expérience de ces rapports. Remarquons

5. Cassirer, Philosophie des formes symboliques, I, p. 28 : «Si l'on pouvait parvenir à une vue systématique des différentes directions de ce mode de l'expression, et à déceler ses traits typiques et communs, ainsi que les gradations particulières et les différences internes de ceux-ci, on accomplirait alors pour l'ensemble de la création spirituelle l'idéal de la « caractéristique universelle » tel que Leibniz l'a formulé pour la connaissance. Nous serions alors en possession d'une espèce de grammaire de la fonction symbolique en tant que telle, qui embrasserait et déterminerait d'une façon générale l'ensemble des expressions et des idiomes particuliers tels que nous les rencontrons dans le langage et dans l'art, dans le mythe et dans la religion. »

6. Cf. pour le problème de l'objectivité, J. Lassègue, «La genèse des concepts mathématiques, entre sciences de la cognition et sciences de la culture », Revue de synthèse, 2003. 
cependant que le fait de renoncer à une démarche intégralement a priori dans la détermination des rapports entre formes symboliques ne condamne pas nécessairement à une simple description empirique de cette détermination, dans la mesure où l'histoire n'est pas un bloc étale mais qu'elle est elle-même interprétable comme composée de moments spécifiques dont la temporalité est qualitativement différente les uns des autres.

Prenons pour exemple la façon dont Cassirer réfléchit à l'avènement de la rationalité scientifique en Grèce classique. Pour lui, l'avènement de la rationalité scientifique et philosophique est un moment de crise du sens qui a consisté avant tout en un divorce avec le monde du mythe, du rite et du langage 7 . Cassirer est donc tout à la fois capable de montrer le caractère essentiellement intriqué du langage, du mythe et du rite et de montrer comment cette intrication a néanmoins pu se dénouer dans le moment historique particulier que représente l'avènement d'une rationalité à la fois scientifique et philosophique. C'est pourquoi il considère également que le monde du mythe, du rite et du langage n'est pas un monde qui serait définitivement dépassé par l'avènement du rationnel puisque ce monde occupe pour toujours le soubassement anthropologique à partir duquel une rationalité scientifique et philosophique peut se développer. Il en veut d'ailleurs pour preuve son projet philosophique personnel, consistant à chercher à réintégrer dans l'orbite du rationnel ce monde archaïque qui en avait été exclu de façon principielle par la science et la philosophie en Grèce classique, en vue de montrer la cohérence globale de l'activité sémiotique en général. Cette tentative de réintégration est elle-même l'indice d'une nouvelle crise, de nature autant politique qu'intellectuelle, à laquelle Cassirer se trouve personnellement confronté dans les années vingt avec la montée de l'idéologie nazie dans la République de Weimar. Deux options lui semblent en effet possibles : soit réintégrer le monde du mythe, du rite et du langage sur un mode rationnel en en faisant des objets de connaissance à part entière, soit reproduire ce monde archaïque par une manipulation généralisée des consciences au moyen de techniques médiatiques appropriées en gommant tout l'itinéraire historique de la rationalité au sein de l'histoire de la culture. On sait combien Cassirer eut à payer d'avoir choisi la première option, lui qui fut contraint à l'exil dès l'arrivée de Hitler au pouvoir.

7. Cassirer, Philosophie des formes symboliques,1929, tome 3, p. 29 : «On comprend très bien que la philosophie, vu sa nature propre et les conditions historiques de sa naissance, n'ait introduit qu'assez tard dans son champ d'investigation l'ensemble des problèmes formels impliqués dans le mythe et dans le langage, après avoir longtemps évité ou repoussé de son seuil ces problèmes plutôt que d'enquêter sur eux. Car le concept de philosophie n'atteint toute sa force et toute sa pureté que là où un dépassement de principe nous fait abandonner la conception du monde exprimée par les concepts de la langue et du mythe [...] C'est sur une voie semblable à celle de la philosophie pure que la connaissance scientifique de la nature arrive à saisir la tâche qui lui est propre. Elle doit elle aussi, pour se trouver elle-même, avoir préalablement accompli la grande scission spirituelle, la krisis de la pensée par laquelle elle se sépare du mythe et du langage. [...] Dans les débuts de la philosophie grecque, les deux problèmes se confondent immédiatement. » 
Il reste toutefois que Cassirer ne thématise pas en propre la nature des rapports qu'entretiennent les formes symboliques entre elles (comme a pu le faire, à sa manière, un Hegel) et que l'on trouve à peine esquissée chez lui l'analyse des crises (au sens qu'il donne à ce terme) qui peuvent parcourir les rapports entre la religion et la science ou entre le langage et la politique. Cela vient sans doute de ses intérêts personnels et de sa formation d'épistémologue néo-kantien, qui l'ont porté à focaliser son attention sur la façon dont la tradition scientifique (en particulier les sciences expérimentales, physique et chimie) pouvait se développer à partir d'un terreau archaïque. Mais la question des rapports entre formes symboliques se retrouve aujourd'hui sous d'autres guises dans les sciences humaines et sociales, que ce soit dans le cadre de la linguistique, de l'anthropologie, de la psychologie culturelle ou de l'histoire des religions. Ce n'est pas le lieu de mesurer comment, dans ce cadre, se trouvent négociés ces différents rapports ${ }^{8}$.

\section{Conclusion}

A partir du moment où l'on considère que la cognition relève d'une interaction ayant l'émergence de valeurs pour objet et qu'elle n'est pas seulement le résultat d'une instanciation de catégories pré-programmées dans des agents préalablement individués, on voit aussi comment intervient la notion de culture dans la cognition. Elle implique un nouveau partage entre l'a priori et l'expérience, que la notion de forme symbolique rend concevable.

\section{Bibliographie}

Cassirer E., Philosophie des formes symboliques, tome 3, La phénoménologie de la connaissance, Editions de minuit, paris, 1972.

Cassirer E., Langage et mythe ; à propos des noms des dieux, Editions de Minuit, Paris, 1973.

Hocart A. M., Kingship Oxford University Press, Oxford, 1927.

Hocart A. M., Social Origins, Watts \& Co, Londres, 1954.

Lassègue J., «La genèse des concepts mathématiques, entre sciences de la cognition et sciences de la culture », Revue de synthèse, $5^{\mathrm{e}}$ série, tome 124, 2004, p. 224-236.

Petitot J., Les catastrophes de la parole, Maloine, Paris, 1985.

de Saussure F., Cours de linguistique générale, Payot, Paris, 1965.

http://formes-symboliques.org

8. C'est précisément le sens de la recherche collective menée au sein du séminaire 'formes symboliques' (http://formes-symboliques.org). 